\title{
Smarandache Curves of the Evolute Curve According to Sabban Frame
}

\author{
Süleyman Şenyurt ${ }^{1 *}$, Yasin Altun ${ }^{1}$
}

\begin{abstract} these curves. Finally, the results are expressed depending on the involute curve. curves.

2010 AMS: 53A04.

\section{${ }^{1}$ Department of Mathematics, Faculty of Science and Arts, Ordu University, Ordu, Turkey.}

*Corresponding author: senyurtsuleyman@hotmail.com

Received: 21 July 2019, Accepted: 23 January 2020, Available online: 25 March 2020
\end{abstract}

The aim of this paper is to define Smarandache curves according to the Sabban frame belonging to the unit Darboux vector of spherical indicatrix curve of the evolute curve. Also, we calculate the geodesic curvatures of

Keywords: Darboux vector, Involute curve, Evolute curve, Geodesic curvature, Sabban frame, Smarandache

\section{Introduction and Preliminaries}

In the theory of curves in the Euclidean space, one of the interesting problems is the characterization of a regular curve. It is known that the shape and size of a regular curve can be determined by using its curvature and torsion. Another approach to the solution of the problem is to consider the relationship between the corresponding Frenet vectors of two curves. For example, involute and evolute curves arise from this relationship. By definition, if the position vector of a curve is composed by the Frenet frame's vectors of another curve, then the curve is called a Smarandache curve [6]. Special Smarandache curves studied by some authors $[1,2,5,6,7,8]$, and related reference therein $[9,10,11]$.

Let $\alpha: I \rightarrow E^{3}$ be a unit speed curve denoted by the moving Frenet apparatus of $\{T, N, B, \kappa, \tau\}$. The Frenet formulae is given by [3]

$$
T^{\prime}(s)=\kappa(s) N(s), N^{\prime}(s)=-\kappa(s) T(s)+\tau(s) B(s), B^{\prime}(s)=-\tau(s) N(s) .
$$

The Darboux vector defined by

$$
W=\tau T+\kappa B .
$$

The unit Darboux vector is given by

$$
C=\sin \omega T+\cos \omega B
$$

where

$$
\sin \omega=\frac{\tau}{\|W\|}, \cos \omega=\frac{\kappa}{\|W\|}, \angle(W, B)=\omega,[12]
$$

Let $\alpha: I \rightarrow \mathbb{E}^{3}$ be a unit speed curve and $\alpha_{1}: I \rightarrow \mathbb{E}^{3}$ be a $C^{2}-$ differentiable curve. If the tangent vector of the curve $\alpha$ is orthogonal to the tangent vector of the $\alpha_{1}$, then $\alpha_{1}$ is called evolute of the $\alpha$.

If the curve $\alpha_{1}$ is evolute of $\alpha$, then we can write,

$$
\alpha_{1}(s)=\alpha(s)+\rho(s) N(s)-\rho(s) \tan (\varphi(s)+c) B(s)
$$




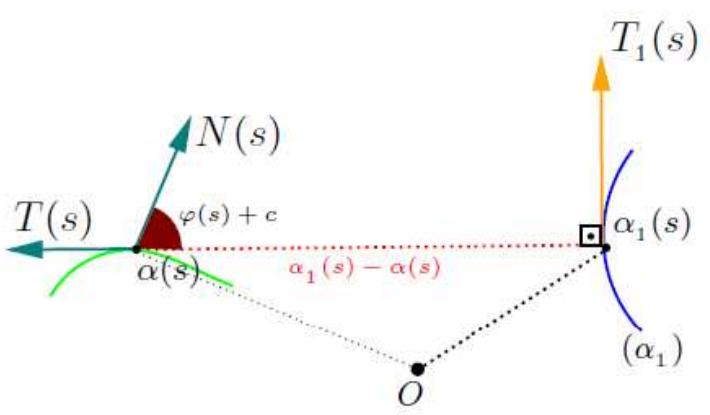

Figure 1.1. Evolute curve

where $c \in R, \rho=\frac{1}{\kappa}$ and $\varphi(s)=\int_{0}^{s} \tau(s) d s$. Also the relations between the Frenet vectors of evolute involute curves are given as [3]

$$
\left\{\begin{array}{l}
T_{1}(s)=\cos (\varphi+c) N(s)-\sin (\varphi+c) B(s) \\
N_{1}(s)=-T(s) \\
B_{1}(s)=\sin (\varphi+c) N+\cos (\varphi+c) B,
\end{array}\right.
$$

and for the curvatures we have

$$
\kappa_{1}(s)=\frac{\kappa^{3} \cos ^{3}(\varphi+c)}{\kappa \tau \sin (\varphi+c)-\kappa^{\prime} \cos (\varphi+c)}, \tau_{1}(s)=\frac{-\kappa^{3} \sin (\varphi+c) \cos ^{2}(\varphi+c)}{\kappa \tau \sin (\varphi+c)-\kappa^{\prime} \cos (\varphi+c)} .
$$

Similarly (1.2), we can write of Darboux vector of evolut curve

$$
W_{1}=\tau_{1} T_{1}+\kappa_{1} B_{1} .
$$

Let $\gamma: I \rightarrow S^{2}$ be a unit speed spherical curve. We can write

$$
\gamma(s)=\gamma(s), t(s)=\gamma^{\prime}(s), d(s)=\gamma(s) \wedge t(s),[5]
$$

here the set $\{\gamma(s), t(s), d(s)\}$ denotes the Sabban frame of $\gamma$ on $S^{2}$. It follows that we also have the equations,

$$
\gamma^{\prime}(s)=t(s), t^{\prime}(s)=-\gamma(s)+\kappa_{g}(s) d(s), d^{\prime}(s)=-\kappa_{g}(s) t(s),[5] .
$$

where $\kappa_{g}$ is the geodesic curvature of the curve $\gamma$ on $S^{2}$ whose curvature is

$$
\kappa_{g}(s)=\left\langle t^{\prime}(s), d(s)\right\rangle[4,5] .
$$

\section{Smarandache curves of the evolute curve according to Sabban frame}

Let $C_{1}$ be the unit Darboux vector of the evolute curve $\alpha_{1}$ and let $\left(C_{1}\right)$ be the unit speed spherical curve on $S^{2}$. Then we can write

$$
C_{1}=\sin \omega_{1} T_{1}+\cos \omega_{1} B_{1}, T_{C_{1}}=\cos \omega_{1} T_{1}-\sin \omega_{1} B_{1}, C_{1} \wedge T_{C_{1}}=N_{1}
$$

where $\angle\left(W_{1}, B_{1}\right)=\omega_{1}$. It follows from the equation (1.6) that of $\left(C_{1}\right)$ are

$$
C_{1}^{\prime}=T_{C_{1}}, T_{C_{1}}^{\prime}=-C_{1}+\frac{\left\|W_{1}\right\|}{\omega_{1}{ }^{\prime}} C_{1} \wedge T_{C_{1}},\left(C_{1} \wedge T_{C_{1}}\right)^{\prime}=-\frac{\left\|W_{1}\right\|}{\omega_{1}{ }^{\prime}} T_{C_{1}} .
$$

From the equation (1.7), we have the following geodesic curvature of $\left(C_{1}\right)$

$$
\kappa_{g}=\left\langle T_{C_{1}}^{\prime}, C_{1} \wedge T_{C_{1}}\right\rangle \Longrightarrow \kappa_{g}=\frac{\left\|W_{1}\right\|}{\omega_{1}^{\prime}} .
$$

Definition 2.1. Let $C_{1}$ be a spherical curve on $S^{2}$ and let $C_{1}, T_{C_{1}}$ be the unit vectors of $\left(C_{1}\right)$. In this case $\beta_{1}(s)$ can be defined by

$$
\beta_{1}(s)=\frac{1}{\sqrt{2}}\left(C_{1}+T_{C_{1}}\right)
$$


In other words, substituting the equation (2.1) into equation (2.3) we write

$$
\beta_{1}(s)=\frac{1}{\sqrt{2}}\left(\left(\sin \omega_{1}+\cos \omega_{1}\right) T_{1}+\left(\cos \omega_{1}-\sin \omega_{1}\right) B_{1}\right) .
$$

Theorem 2.2. The geodesic curvature of $\beta_{1}(s)$-Smarandache curve is given by

$$
\kappa_{g}^{\beta_{1}}=\frac{1}{\left(2+\frac{1}{\eta^{2}}\right)^{\frac{5}{2}}}\left(\frac{1}{\eta} \bar{\lambda}_{1}-\frac{1}{\eta} \bar{\lambda}_{2}+2 \bar{\lambda}_{3}\right)
$$

where

$$
\eta=\frac{\kappa \tau^{2} \sin (\varphi+c)-\kappa^{\prime} \tau \cos (\varphi+c)}{\kappa^{3} \cos ^{2}(\varphi+c)}, \bar{\lambda}_{1}=-2-\frac{1}{\eta^{2}}+\frac{1}{\eta^{\prime}} \frac{1}{\eta}, \bar{\lambda}_{2}=-2-3 \frac{1}{\eta^{2}}-\frac{1}{\eta^{4}}-\frac{1}{\eta^{\prime}} \frac{1}{\eta}, \bar{\lambda}_{3}=2 \frac{1}{\eta}+\frac{1}{\eta^{3}}+\frac{1}{\eta^{\prime}} .
$$

Proof: By differentiating (2.3) we can write

$$
T_{\beta_{1}}=\frac{\omega_{1}^{\prime}\left(\cos \omega_{1}-\sin \omega_{1}\right)}{\sqrt{2 \omega_{1}^{\prime 2}+\left\|W_{1}\right\|^{2}}} T_{1}+\frac{\left\|W_{1}\right\|}{\sqrt{2 \omega_{1}^{\prime 2}+\left\|W_{1}\right\|^{2}}} N_{1}-\frac{\omega_{1}^{\prime}\left(\cos \omega_{1}+\sin \omega_{1}\right)}{\sqrt{2 \omega_{1}^{\prime 2}+\left\|W_{1}\right\|^{2}}} B_{1} .
$$

Considering the equations (2.4) and (2.5) we get

$$
\beta_{1} \wedge T_{\beta_{1}}=\frac{\left\|W_{1}\right\|\left(\cos \omega_{1}+\sin \omega_{1}\right)}{\sqrt{2\left\|W_{1}\right\|^{2}+4\left(\omega_{1}^{\prime}\right)^{2}}} T_{1}-\frac{\omega_{1}^{\prime}}{\sqrt{2\left\|W_{1}\right\|^{2}+4\left(\omega_{1}^{\prime}\right)^{2}}} N_{1}+\frac{\left\|W_{1}\right\|\left(\cos \omega_{1}+\sin \omega_{1}\right)}{\sqrt{2\left\|W_{1}\right\|^{2}+4\left(\omega_{1}^{\prime}\right)^{2}}} B_{1} .
$$

it follows by differentiating (2.5), with the coefficients

$\aleph_{1}=-2-\left(\frac{\left\|W_{1}\right\|}{\omega_{1}{ }^{\prime}}\right)^{2}+\left(\frac{\left\|W_{1}\right\|}{\omega_{1}{ }^{\prime}}\right)^{\prime}\left(\frac{\left\|W_{1}\right\|}{\omega_{1}{ }^{\prime}}\right), \aleph_{2}=-2-3\left(\frac{\left\|W_{1}\right\|}{\omega_{1}{ }^{\prime}}\right)^{2}-\left(\frac{\left\|W_{1}\right\|}{\omega_{1}{ }^{\prime}}\right)^{4}-\left(\frac{\left\|W_{1}\right\|}{\omega_{1}{ }^{\prime}}\right)^{\prime}\left(\frac{\left\|W_{1}\right\|}{\omega_{1}{ }^{\prime}}\right), \aleph_{3}=2\left(\frac{\left\|W_{1}\right\|}{\omega_{1}{ }^{\prime}}\right)+\left(\frac{\left\|W_{1}\right\|}{\omega_{1}{ }^{\prime}}\right)^{3}+\left(\frac{\left\|W_{1}\right\|}{\omega_{1}{ }^{\prime}}\right)^{\prime}$

we can find out,

$$
T_{\beta_{1}}^{\prime}=\frac{\left(\omega_{1}^{\prime}\right)^{4} \sqrt{2}\left(\aleph_{1} \sin \omega_{1}+\aleph_{2} \cos \omega_{1}\right)}{\left(\left\|W_{1}\right\|^{2}+\left(\omega_{1}^{\prime}\right)^{2}\right)^{2}} T_{1}+\frac{\aleph_{3}\left(\omega_{1}^{\prime}\right)^{4} \sqrt{2}}{\left(\left\|W_{1}\right\|^{2}+\left(\omega_{1}^{\prime}\right)^{2}\right)^{2}} N_{1}+\frac{\left(\omega_{1}^{\prime}\right)^{4} \sqrt{2}\left(\aleph_{1} \cos \omega_{1}-\aleph_{2} \sin \omega_{1}\right)}{\left(\left\|W_{1}\right\|^{2}+\left(\omega_{1}^{\prime}\right)^{2}\right)^{2}} B_{1} .
$$

From the equation (2.6) and (2.8), geodesic curvature $\kappa_{g}^{\beta_{1}}$ for the evolute curve $\beta_{1}$ is

$$
\kappa_{g}^{\beta_{1}}=\left\langle T_{\beta_{1}}^{\prime}, \beta_{1} \wedge T_{\beta_{1}}\right\rangle=\frac{1}{\left(2+\left(\frac{\left\|W_{1}\right\|}{\omega_{1}^{\prime}}\right)^{2}\right)^{\frac{5}{2}}}\left(\frac{\left\|W_{1}\right\|}{\omega_{1}^{\prime}} \aleph_{1}-\frac{\left\|W_{1}\right\|}{\omega_{1}^{\prime}} \aleph_{2}+2 \aleph_{3}\right) .
$$

From the equation (1.3) and (1.4) Sabban apparatus of the $\beta_{1}$-Smarandache curve for involute curves are

$$
\begin{aligned}
\beta_{1}(s) & =\frac{1}{\sqrt{2}}(N+B), T_{\beta_{1}}=\frac{1}{\sqrt{1+2 \eta^{2}}}(-T+\eta N-\eta B), \\
\beta_{1} \wedge T_{\beta_{1}} & =\frac{1}{\sqrt{2+4 \eta^{2}}}(-2 \eta T-\eta N+B), T_{\beta_{1}}^{\prime}=\frac{\sqrt{2}}{\left(2 \eta^{2}+1\right)^{2}}\left(-\eta^{4} \bar{\lambda}_{3} T+\eta^{4} \bar{\lambda}_{2} N+\eta^{4} \bar{\lambda}_{1} B\right),
\end{aligned}
$$

hence the proof is completed.

Definition 2.3. Let $C_{1}$ be a spherical curve on $S^{2}$ and let $C_{1}, C_{1} \wedge T_{C_{1}}$ be the unit vectors of $\left(C_{1}\right)$. In this case $\beta_{2}(s)$ can be defined by

$$
\beta_{2}(s)=\frac{1}{\sqrt{2}}\left(C_{1}+C_{1} \wedge T_{C_{1}}\right) .
$$

From the equation (1.3), (1.4) and (2.1) we can write

$$
\beta_{2}=\frac{1}{\sqrt{2}}(-T+B) .
$$

Theorem 2.4. The geodesic curvature of $\beta_{2}(s)$-Smarandache curve is given by

$$
\kappa_{g}^{\beta_{2}}=\frac{1+\eta}{\eta-1} .
$$


Proof: By differentiating (2.10), we can write

$$
T_{\beta_{2}}=N
$$

Considering the equations (2.10) and (2.11) it is easily seen that

$$
\beta_{2} \wedge T_{\beta_{2}}=\frac{1}{\sqrt{2}}(-T-B)
$$

and then differentiating (2.11) we can write

$$
T_{\beta_{2}}^{\prime}=\frac{\sqrt{2}}{\eta-1}(-T-\eta B) .
$$

It follows that $\kappa_{g}^{\beta_{2}}$ geodesic curvature for $\beta_{2}$ is

$$
\kappa_{g}^{\beta_{2}}=\frac{1+\eta}{\eta-1} .
$$

Definition 2.5. Let $C_{1}$ be a spherical curve on $S^{2}$ and let $T_{C_{1}}, C_{1} \wedge T_{C_{1}}$ be the unit vectors of $\left(C_{1}\right)$. In this case $\beta_{3}(s)$ can be defined by

$$
\beta_{3}(s)=\frac{1}{\sqrt{2}}\left(T_{C_{1}}+C_{1} \wedge T_{C_{1}}\right)
$$

From the equations (2.1), (1.3) and (1.4) we can write

$$
\beta_{3}=\frac{1}{\sqrt{2}}(-T+N) \text {. }
$$

Theorem 2.6. The geodesic curvature of $\beta_{3}(s)$-Smarandache curve is given by

$$
\kappa_{g}^{\beta_{3}}=\frac{1}{\left(2+\frac{1}{\eta^{2}}\right)^{\frac{5}{2}}}\left(2 \frac{1}{\eta} \bar{\sigma}_{1}-\bar{\sigma}_{2}+\bar{\sigma}_{3}\right) .
$$

Proof: By differentiating (2.13) we have

$$
T_{\beta_{3}}=\frac{1}{\sqrt{2+\eta^{2}}}(-T-N-\eta B) .
$$

Considering the equations (2.13) and (2.15) it is easily seen that

$$
\beta_{3} \wedge T_{\beta_{3}}=\frac{1}{\sqrt{2 \eta^{2}+4}}(-\eta T-\eta N+2 B) .
$$

By differentiating (2.15) with the coefficients

$$
\bar{\sigma}_{1}=\frac{1}{\eta}+2 \frac{1}{\eta^{3}}+2 \frac{1}{\eta^{\prime}} \frac{1}{\eta}, \bar{\sigma}_{2}=-1-3 \frac{1}{\eta^{2}}-2 \frac{1}{\eta^{4}}-\frac{1}{\eta^{\prime}}, \bar{\sigma}_{3}=-\frac{1}{\eta^{2}}-2 \frac{1}{\eta^{4}}+\frac{1}{\eta^{\prime}}
$$

we get

$$
T_{\beta_{3}}^{\prime}=\frac{\sqrt{2}}{\left(\eta^{2}+2\right)^{2}}\left(-\eta^{4} \bar{\sigma}_{3} T+\eta^{4} \bar{\sigma}_{2} N+\eta^{4} \bar{\sigma}_{1} B\right) .
$$

By this way, geodesic curvature $\kappa_{g}^{\beta_{3}}$ for the involute curve of $\beta_{3}$ is given by

$$
\kappa_{g}^{\beta_{3}}=\frac{1}{\left(2+\frac{1}{\eta^{2}}\right)^{\frac{5}{2}}}\left(2 \frac{1}{\eta} \bar{\sigma}_{1}-\bar{\sigma}_{2}+\bar{\sigma}_{3}\right) .
$$

Definition 2.7. Let $C_{1}$ be a spherical curve on $S^{2}$ and let $T_{C_{1}}, C_{1} \wedge T_{C_{1}}$ be the unit vectors of $\left(C_{1}\right)$. In this case $\beta_{4}(s)$ can be defined by

$$
\beta_{4}(s)=\frac{1}{\sqrt{3}}\left(C_{1}+T_{C_{1}}+C_{1} \wedge T_{C_{1}}\right)
$$


From the equations (1.3), (1.4) and (2.1) we can write that

$$
\beta_{4}(s)=\frac{1}{\sqrt{3}}(-T+N+B) .
$$

Theorem 2.8. The geodesic curvature of $\beta_{4}(s)$-Smarandache curve is given by

$$
\kappa_{g}^{\beta_{4}}=\frac{\left(2 \frac{1}{\eta}-1\right) \bar{\psi}_{1}+\left(-1-\frac{1}{\eta}\right) \bar{\psi}_{2}+\left(2-\frac{1}{\eta}\right) \bar{\psi}_{3}}{4 \sqrt{2}\left(1-\frac{1}{\eta}+\frac{1}{\eta^{2}}\right)^{\frac{5}{2}}} .
$$

Proof: By differentiating (2.18), we get

$$
T_{\beta_{4}}=\frac{1}{\sqrt{2\left(1-\eta+\eta^{2}\right)}}(-T+(\eta-1) N-\eta B) .
$$

Considering the equations (2.18) and (2.19) it is easily seen that

$$
\beta_{4} \wedge T_{\beta_{4}}=\frac{1-2 \eta}{\sqrt{6-6 \eta+6 \eta^{2}}} T-\frac{\eta+1}{\sqrt{6-6 \eta+6 \eta^{2}}} N+\frac{2-\eta}{\sqrt{6-6 \eta+6 \eta^{2}}} B .
$$

By differentiating (2.19), with the coefficients

$\bar{\psi}_{1}=-2+4 \frac{1}{\eta}-4 \frac{1}{\eta^{2}}+2 \frac{1}{\eta^{3}}+2 \frac{1}{\eta^{\prime}}\left(2 \frac{1}{\eta}-1\right), \bar{\psi}_{2}=-2+2 \frac{1}{\eta}-4 \frac{1}{\eta^{2}}+2 \frac{1}{\eta^{3}}-2 \frac{1}{\eta^{4}}-\frac{1}{\eta^{\prime}}\left(1+\frac{1}{\eta}\right), \bar{\psi}_{3}=2 \frac{1}{\eta}-4 \frac{1}{\eta^{2}}+4 \frac{1}{\eta^{3}}-2 \frac{1}{\eta^{4}}+\frac{1}{\eta^{\prime}}\left(2-\frac{1}{\eta}\right)$ we can write that

$$
T_{\beta_{4}}^{\prime}=\frac{1}{4\left(1+\eta+\eta^{2}\right)^{2}}\left(-\bar{\psi}_{3} \eta^{4} T+\bar{\psi}_{2} \eta^{4} N+\bar{\psi}_{1} \eta^{4} B\right)
$$

then we get geodesic curvature $\kappa_{g}^{\beta_{4}}$, for the involute curve $\beta_{4}\left(s_{\beta_{4}}\right)$ as

$$
\kappa_{g}^{\beta_{4}}=\frac{\left(2 \frac{1}{\eta}-1\right) \bar{\psi}_{1}+\left(-1-\frac{1}{\eta}\right) \bar{\psi}_{2}+\left(2-\frac{1}{\eta}\right) \bar{\psi}_{3}}{4 \sqrt{2}\left(1-\frac{1}{\eta}+\frac{1}{\eta^{2}}\right)^{\frac{5}{2}}} .
$$

Example. Let us consider the unit speed spherical curve

$$
\alpha(t)=\left(\frac{2}{5} \sin (2 t)-\frac{1}{40} \sin (8 t),-\frac{2}{5} \cos (2 t)+\frac{1}{40} \cos (8 t), \frac{4}{15} \sin (3 t)\right)
$$

and evolute of this curve,

$$
\begin{aligned}
\alpha_{1}(t)= & \left(\frac{1}{40} \frac{16 \sin (2 t) \sin (3 t) \cos (1)-\sin (8 t) \sin (3 t) \cos (1)+8 \cos (5 t) \cos (1)}{\sin (3 t) \cos (1)}-\frac{1}{40} \frac{8 \sin (1) \sin (2 t)-2 \sin (1) \sin (8 t)}{\sin (3 t) \cos (1)}\right. \\
& -\frac{1}{40} \frac{16 \cos (2 t) \sin (3 t) \cos (1)-\cos (8 t) \sin (3 t) \cos (1)}{\sin (3 t) \cos (1)}+\frac{1}{40} \frac{8 \sin (5 t) \cos (1)-8 \sin (1) \cos (2 t)-2 \sin (1) \cos (8 t)}{\sin (3 t) \cos (1)} \\
& \left.\frac{1}{60} \frac{7 \cos (1)-16 \cos (1)(\cos (3 t))^{2}+12 \sin (1) \sin (3 t)}{\sin (3 t) \cos (1)}\right)
\end{aligned}
$$

and Frenet vectors of the evolute curve $\alpha_{1}$,

$$
\begin{aligned}
T_{1}= & \left(\frac{64}{5} \cos (1)(\cos (t))^{5}-16 \cos (1)(\cos (t))^{3}+4 \cos (1) \cos (t)+\frac{128}{5} \sin (1) \sin (t)(\cos (t))^{7}-\frac{192}{5} \sin (1) \sin (t)(\cos (t))^{5}\right. \\
& +16 \sin (1) \sin (t)(\cos (t))^{3}, \frac{64}{5} \cos (1) \sin (t)(\cos (t))^{4}-\frac{48}{5} \sin (t)(\cos (t))^{2} \cos (1)+\frac{4}{5} \cos (1) \sin (t)+\frac{24}{5} \sin (1)(\cos (t))^{2}+\frac{3}{5} \sin (1) \\
& \left.-\frac{128}{5} \sin (1)(\cos (t))^{8}+\frac{256}{5} \sin (1)(\cos (t))^{6}-32 \sin (1)(\cos (t))^{4},-\frac{3}{5} \cos (1)-\frac{16}{5} \sin (1) \sin (t)(\cos (t))^{2}+\frac{4}{5} \sin (1) \sin (t)\right) \\
N_{1}= & \left(-\frac{4}{5} \cos (2 t)+\frac{1}{5} \cos (8 t),-\frac{4}{5} \sin (2 t)+\frac{1}{5} \sin (8 t),-\frac{4}{5} \cos (3 t)\right) \\
B_{1}= & \left(-\frac{128}{5} \sin (t)(\cos (t))^{7} \cos (1)+\frac{192}{5} \sin (t)(\cos (t))^{5} \cos (1)-16 \sin (t)(\cos (t))^{3} \cos (1)+\frac{64}{5}(\cos (t))^{5} \sin (1)\right. \\
& -16(\cos (t))^{3} \sin (1)+4 \cos (t) \sin (1)-\frac{24}{5}(\cos (t))^{2} \cos (1)-3 / 5 \cos (1)+\frac{128}{5}(\cos (t))^{8} \cos (1) \\
& -\frac{256}{5} \cos (1)(\cos (t))^{6}+32(\cos (t))^{4} \cos (1)+\frac{64}{5} \sin (t)(\cos (t))^{4} \sin (1) \\
& \left.-\frac{48}{5} \sin (1) \sin (t)(\cos (t))^{2}+4 / 5 \sin (1) \sin (t), \frac{16}{5} \sin (t)(\cos (t))^{2} \cos (1)-4 / 5 \cos (1) \sin (t)-3 / 5 \sin (1)\right)
\end{aligned}
$$


where $c=1$ and $t=0$ to $2 \pi$.

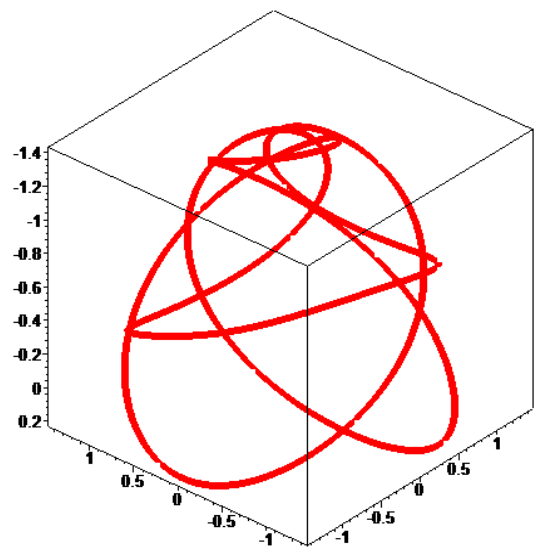

Figure 2.1. $C_{1} T_{C_{1}}$-Smarandache curve $\left(\beta_{1}\right.$-curve $)$

$$
\begin{aligned}
\beta_{1}(t)= & \left(-\frac{128}{5} \sin (t)(\cos (t))^{7}+\frac{192}{5} \sin (t)(\cos (t))^{5}-16 \sin (t)(\cos (t))^{3}+\frac{64}{5}(\cos (t))^{5}-16(\cos (t))^{3}+4 \cos (t)\right. \\
& -\frac{24}{5}(\cos (t))^{2}-\frac{3}{5}+\frac{128}{5}(\cos (t))^{8}-\frac{256}{5}(\cos (t))^{6}+32(\cos (t))^{4}+\frac{64}{5} \sin (t)(\cos (t))^{4}-\frac{48}{5} \sin (t)(\cos (t))^{2}+\frac{4}{5} \sin (t), \\
& \left.\frac{16}{5} \sin (t)(\cos (t))^{2}-\frac{4}{5} \sin (t)-\frac{3}{5}\right)
\end{aligned}
$$

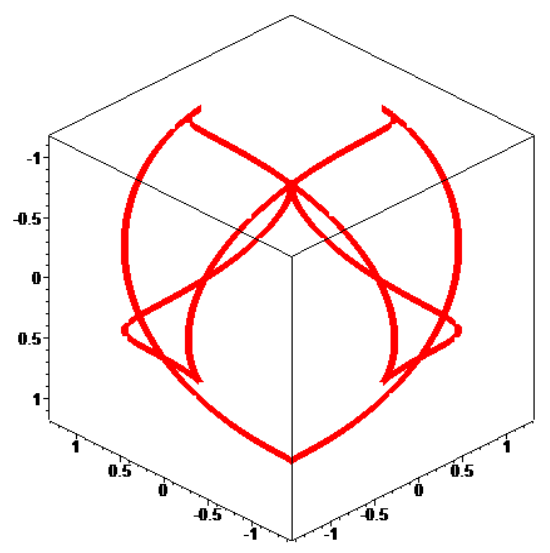

Figure 2.2. $C_{1}\left(C_{1} \wedge T_{C_{1}}\right)$-Smarandache curve $\left(\beta_{2}\right.$-curve $)$

$$
\begin{aligned}
\beta_{2}(t)= & \left(-8(\cos (t))^{2}+1+\frac{128}{5}(\cos (t))^{8}-\frac{256}{5}(\cos (t))^{6}+32(\cos (t))^{4}-\frac{128}{5} \sin (t)(\cos (t))^{7}+\frac{192}{5} \sin (t)(\cos (t))^{5}-16 \sin (t)(\cos (t))^{3},\right. \\
& -\frac{16}{5} \sin (t) \cos (t)+\frac{128}{5} \sin (t)(\cos (t))^{7}-\frac{192}{5} \sin (t)(\cos (t))^{5}+16 \sin (t)(\cos (t))^{3}-\frac{24}{5}(\cos (t))^{2}-\frac{3}{5}+\frac{128}{5}(\cos (t))^{8} \\
& \left.-\frac{256}{5}(\cos (t))^{6}+32(\cos (t))^{4},-\frac{16}{5}(\cos (t))^{3}+\frac{12}{5} \cos (t)+\frac{16}{5} \sin (t)(\cos (t))^{2}-\frac{4}{5} \sin (t)\right)
\end{aligned}
$$




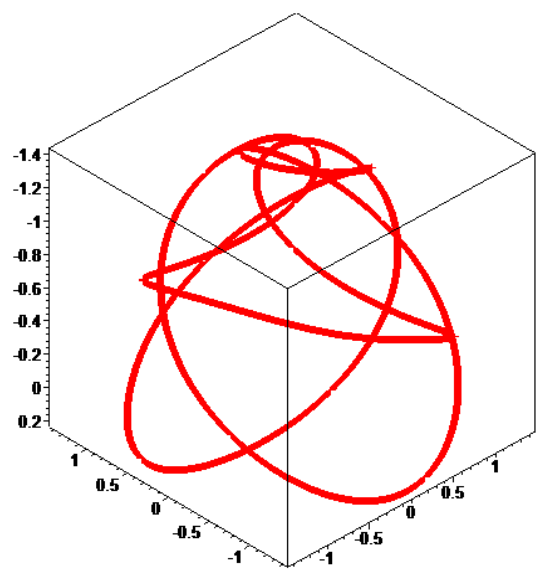

Figure 2.3. $T_{C_{1}}\left(C_{1} \wedge T_{C_{1}}\right)$-Smarandache curve $\left(\beta_{3}\right.$-curve $)$

$$
\begin{aligned}
\beta_{3}(t)= & \left(-8(\cos (t))^{2}+1+\frac{128}{5}(\cos (t))^{8}-\frac{256}{5}(\cos (t))^{6}+32(\cos (t))^{4}+\frac{64}{5}(\cos (t))^{5}-16(\cos (t))^{3}+4 \cos (t),\right. \\
& -\frac{16}{5} \sin (t) \cos (t)+\frac{128}{5} \sin (t)(\cos (t))^{7}-\frac{192}{5} \sin (t)(\cos (t))^{5}+16 \sin (t)(\cos (t))^{3}+\frac{64}{5} \sin (t)(\cos (t))^{4} \\
& \left.-\frac{48}{5} \sin (t)(\cos (t))^{2}+\frac{4}{5} \sin (t),-\frac{16}{5}(\cos (t))^{3}+\frac{12}{5} \cos (t)-\frac{3}{5}\right)
\end{aligned}
$$

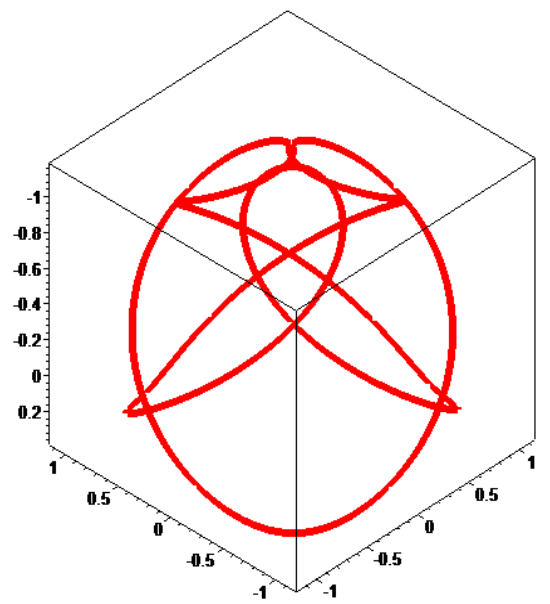

Figure 2.4. $C_{1} T_{C_{1}}\left(C_{1} \wedge T_{C_{1}}\right)$-Smarandache curve $\left(\beta_{4}\right.$-curve $)$

$$
\begin{aligned}
\beta_{4}(t)= & \left(-\frac{256}{15} \sin (t)(\cos (t))^{7}+\frac{128}{5} \sin (t)(\cos (t))^{5}-\frac{32}{3} \sin (t)(\cos (t))^{3}+\frac{128}{15}(\cos (t))^{5}-\frac{32}{3}(\cos (t))^{3}+\frac{8}{3} \cos (t)-\frac{16}{3}(\cos (t))^{2}+\frac{2}{3}\right. \\
& +\frac{256}{15}(\cos (t))^{8}-\frac{512}{15}(\cos (t))^{6}+\frac{64}{3}(\cos (t))^{4},-\frac{16}{5}(\cos (t))^{2}-\frac{2}{5}+\frac{256}{15}(\cos (t))^{8}-\frac{512}{15}(\cos (t))^{6}+\frac{64}{3}(\cos (t))^{4} \\
& +\frac{128}{15} \sin (t)(\cos (t))^{4}-\frac{32}{5} \sin (t)(\cos (t))^{2}+\frac{8}{15} \sin (t)-\frac{32}{15} \sin (t) \cos (t)+\frac{256}{15} \sin (t)(\cos (t))^{7}-\frac{128}{5} \sin (t)(\cos (t))^{5} \\
& \left.+\frac{32}{3} \sin (t)(\cos (t))^{3}, \frac{32}{15} \sin (t)(\cos (t))^{2}-\frac{8}{15} \sin (t)-\frac{2}{5}-\frac{32}{15}(\cos (t))^{3}+\frac{8}{5} \cos (t)\right)
\end{aligned}
$$




\section{References}

[1] A.T. Ali, Special Smarandache Curves in the Euclidian Space, International J.Math. Combin., 2, (2010), 30-36.

[2] A. Çalışkan, S. Şenyurt, Smarandache Curves In Terms of Sabban Frame of Spherical Indicatrix Curves, Gen. Math. Notes, 31(2), (2015), 1-15.

[3] A. Sabuncuoğlu, Differential Geometry, Nobel Publications, 2006.

[4] J. Koenderink, Solid Shape, MIT Press, Cambridge, MA, 1990.

[5] K. Taşköprü, M. Tosun, Smarandache Curves on $S^{2}$, Bol. Soc. Paran. Mat. 32(1), (2014), 51-59.

[6] M. Turgut, S. Yılmaz, Smarandache Curves in Minkowski space-time, International J.Math. Combin., 3, (2008), 51-55.

[7] S. Şenyurt, C. Cevahir, Y. Altun, On Spatial Quaternionic Involute Curve A New View, Adv. Appl. Clifford Algebras, 27(2), (2017), 1815-1824.

[8] S. Şenyurt, C. Cevahir, Y. Altun, H. Kocayiğit, On the Sabban frame belonging to involute-evolute curves, Thermal Science, 23, (2019), 413-425.

[9] K. E. Özen, M. Tosun, M. Akyiğit, Siacci's theorem according to Darboux frame, Analele Universitatii Ovidius Constanta-Seria Matematica, 25(3), (2017), 155-165. DOI:10.1515/auom-2017-0042.

[10] K. E. Özen, F. S. Dündar, M. Tosun, An alternative approach to jerk in motion along a space curve with applications, Journal of Theoretical and Applied Mechanics, 57(2), (2019), 435-444. DOI:10.15632/jtam-pl/104595.

[11] K. E. Özen, M. Güner, M. Tosun, A note on the acceleration and jerk in motion along a space curve, Analele Universitatii Ovidius Constanta-Seria Matematica, 28(1), (2020), 151-164. DOI:10.2478/auom-2020-0011.

[12] W. Fenchel, On The Differential Geometry of Closed Space Curves, Bulletin of the American Mathematical Society, 57, (1951), 44-54. 